\title{
XXVI. On the theory of the compressibility of the matter composing the nucleus of the earth, as confirmed by what is known of the ellipticities of the planets
}

\section{Rev. J. Challis}

To cite this article: Rev. J. Challis (1831) XXVI. On the theory of the compressibility of the matter composing the nucleus of the earth, as confirmed by what is known of the ellipticities of the planets, Philosophical Magazine Series 2, 10:57, 200-204, DOI: $10.1080 / 14786443108675518$

To link to this article: http://dx.doi.org/10.1080/14786443108675518

$$
\text { 曲 Published online: } 14 \text { Jul } 2009 .
$$

Submit your article to this journal ¿

$$
\text { Џlll Article views: } 1
$$




\section{[ 200$]$}

XXVI. On the Theory of the Compressibility of the Matter composing the Nucleus of the Earth, as confirmed by what is known of the Ellipticities of the Planets. By the Rev. J. Challis, Fellow of the Cambridge Philosophical Society*.

R.YOUNG suggested that the increase of density towards the centre of the earth, might be owing to the compressibility of the material of which it is composed. Laplace, adopting the suggestion, obtained, in an addition to a Memoir on the Figure of the Earth (Mém. Acad.Scien. An. 1818) the law of the increase of density in proceeding from the surface to the centre, on the suppositions that the relation between the pressure $(p)$ and density $(\rho)$ is expressed by $p=k^{\circ}\left(\frac{\rho^{2}}{\delta^{2}}-1\right)$, ( $\delta$ being the density at the surface), and that the chemical composition of the nucleus of the earth is the same throughout. He found that on these suppositions the requisite degree of compressibility, and the proportion of the density at the surface to the mean density, were not by any means at variance with what we know on these points by experience. The cause assigned in this theory for the increase of density towards the centre, and the relation between $p$ and $\varrho$, are of so simple a nature, that I have been induced to inquire how far the theory is confirmed by what is known of the ellipticities of the planets.

I here repeat the investigation of Laplace, modified for the purposes I have in view. Suppose the mass of the planet to be spherical. If $r$ be any distance from its centre, and $\rho=$ $\Phi(r)$, the attraction on a particle of the mass at a distance $\mathrm{R}$ from the centre is $\frac{\int 4 \pi r^{2} \phi(r) d r}{\mathrm{R}^{2}}$, the integral being taken from $r=0$ to $r=\mathrm{R}$. To express this force in the manner in which terrestrial gravity is usually expressed, let $M=$ the earth's mass, $a=$ its radius, and $g=32 \frac{1}{6}$ feet, the measure of the accelerative force of gravity at the earth's surface: then $\frac{g a^{2}}{\mathrm{M}} \cdot \frac{f 4 \pi r^{2} \phi(r) d r}{\mathrm{R}^{2}}$, is the force expressed as required. Suppose that $\int r^{2} d r \phi(r)=\psi(r)$. Then attraction (A) $=\frac{4 \pi g a^{2}}{\mathrm{M}} \times \frac{\psi(\mathrm{R})-\psi(0)}{\mathrm{R}^{2}}$. Now $-d p=\mathrm{A}_{\boldsymbol{g}} d \mathrm{R}$; and $-d p=-\frac{2 k^{2}}{\delta^{2}} \rho d \rho$.

$$
\text { Therefore } \frac{4 \pi g a^{2} \rho}{\mathrm{M}} \cdot \frac{\psi(\mathrm{R})-\psi(0)}{\mathrm{R}^{2}}=-\frac{2 k^{2} \rho d \rho}{\delta^{2} d \mathrm{R}} \text {. }
$$

* Communicated by the Author. 
Rev. J. Challis on the Ellipticities of the Planets. 201

Hence $\psi(R)-\psi(0)+\frac{2 k^{2} M}{4 \pi g a^{2} \delta^{2}} \cdot \frac{R^{3} d \rho}{d \mathbf{R}}=0$; and differentiating, $\mathrm{R}^{2} \Phi(\mathrm{R})+\frac{k^{2} \mathrm{M}}{2 \pi g a^{2} \delta^{2}}\left(\frac{\mathrm{R}^{2} d^{2} \rho}{d \mathrm{R}^{2}}+\frac{2 \mathrm{R} d}{d \mathrm{R}}\right)=\mathbf{0}$. Hence finally, $\frac{d^{2} \mathrm{R} \rho}{d \mathrm{R}^{2}}+q^{2} \mathrm{R} \rho=0, q^{2}$ being $\frac{2 \pi g a^{2} \delta^{2}}{k^{2} \mathrm{M}}$. The integral of this gives $\frac{\rho}{\Delta}=\frac{\sin q \mathrm{R}}{q \mathrm{R}}, \Delta$ being the value of $g$ at the centre. Legendre has calculated (Mém. Acad.Scien. An. 1789), that according to this law of density, if $c=$ the earth's radius, and $q c=\frac{2 \pi}{3}$, the ellipticity would be $\frac{1}{269}$; and if $q c=\pi$, the ellipticity would be $\frac{1}{379}$. But it is plain from the nature of solid substances that $q c$ cannot be so great as $\pi$, for then $\varrho$ would be $=0$. In proportion as the value of $q \mathrm{R}$ is near to $\pi$, the density is small, and decreases rapidly as $R$ increases: and because solid substances do not admit of this rapid change of density, and at their surfaces possess a certain limited density, therefore it is probable that we shall not be far wrong by assuming $q c=\frac{5 \pi}{6}$. In fact, this value gives an ellipticity of $\frac{1}{306}$ to the earth, which is very near the experimental determination.

If now we calculate the attractive force of Jupiter at his equator, by means of the period of his fourth satellite, and the law of the inverse square of the distance, we shall find that the ratio of the centrifugal force at his equator to this force is $\frac{1}{11 \cdot 95}$. But because the law of the inverse square does not accurately hold by reason of the planet's spheroidal shape, this value requires a correction. When the correction has been made according to the formula given in the Méc. Céleste, liv. iii. art. 35, the ratio becomes $\frac{1}{12 \cdot 348}$. The ratio of the centrifugal force to gravity at the equator of Saturn, calculated from the period ( $79 \cdot 33$ days) and mean distance (64.36 equatorial radii) of the extreme satellite, and from the time $\left(10^{\mathrm{h}} 15^{\prime}\right)$ of Saturn's rotation, will be found to be $\frac{1}{7 \cdot 76}$. Correcting as before, the ratio becomes $\frac{1}{8}$ nearly. CalcuN.S. Vol. 10. No. 57. Sept. 1831 . $2 \mathrm{D}$ lating 
lating now the ellipticities of Jupiter and Saturn by Clairaut's Theorem, on the supposition that $q c=\frac{5 \pi}{6}$, we shall find for Jupiter $\frac{1}{13 \cdot 1}$, and for Saturn $\frac{1}{8 \cdot 4}$. The measurements of Professor Struve determine the ellipticity of Jupiter to be $\frac{1}{13 \cdot 71}$, and Herschel obtained $\frac{1}{11}$ for the ellipticity of Saturn. It must be observed that the above calculations take into account only the first power of the ellipticity, and therefore cannot be very accurate with respect to Jupiter and Saturn, the ellipticities of which are not very small. We may, however, affirm that the ellipticity of Jupiter accords very well with the theory we are considering. 'That of Saturn is considerably less than what the theory gives. Herschel remarked an anomaly in the shape of this planet, which, however, subsequent observations have not yet confirmed; viz. that the equatorial diameter was not so large as a diameter about midway between the equatorial and the polar. It would seem, if this be true, that some cause has operated to compress the parts about the equator. The same cause would account for an ellipticity less than what our theory requires. Possibly the rings may have something to do with this.

Venus, Mercury, and the Sun, in as much as they possess no ellipticity discoverable by instruments, do not contradict the theory. But Mars forms an exception. Its ellipticity is ascertained by observation to be $\frac{1}{16}$; whereas the ratio of the centrifugal force to gravity at its equator, which ratio differs little from the ellipticity that the theory gives, will be found to be $\frac{1}{254}$, by taking $\cdot 1386$ for the ratio of its mass to that of the earth, $\cdot 1294$ the ratio of the volumes, and $24 \cdot 67$ hours the period of its rotation. The great ellipticity of this planet, considering the time of its rotation and its small size, is remarkable, and seems not to be in accordance with Clairaut's Theorem, unless we suppose the gravity of the planet to diminish in passing from the equator to the pole.

If the cause assigned in this theory be sufficient to account for the increase of the density of a planet towards its centre, then on the supposition that the nuclei of the planets are all as to chemical composition homogeneous, and are similarly constituted, though of different mean densities, the equation $q^{c}=\frac{5 \pi}{6}$ ought to be nearly true for all, since it is nearly 
true for the earth. Let us see what will follow from supposing this equation to be generally true. If the mean density of the earth be $d$, then $\mathrm{M}=\frac{4 \pi \alpha^{3} d}{3}$, and $q^{2}=\frac{3 g \delta^{2}}{2 k^{2} a d}$. Now the velocity $(V)$ of propagation in a medium in which $p=k^{2}\left(\frac{\rho^{2}}{\delta^{2}}-1\right)$, is $\frac{k}{\delta} \sqrt{2 \delta}$, where the density is $\delta$. This may be shown by a separate consideration of this particular case, or be inferred from a general proposition respecting the velocity of propagation, which I gave in the Phil. Mag. and Annals of Philosophy, for May 1830*, where it was proved that if $p+\mathrm{C}=a^{a} e^{1+n}, \mathrm{~V}$ may be found from the equation $\mathrm{V}^{2}-a^{2} \rho^{n}(1+n)=0$. We have then, $\frac{\mathrm{V}^{2}}{2 \delta}=\frac{k^{2}}{\delta^{2}} ; q^{2}=$ $\frac{3 g \delta}{\mathrm{V}^{2} a d}$; and $q^{2} c^{2}$, or $\frac{25 \pi^{2}}{36}=\frac{3 g c^{2} \delta}{\mathrm{V}^{2} a d}$. Now Laplace has calculated that according to this theory the ratio of the mean density to the density at the surface of the earth is 2.42 ; and according to our supposition the same ratio holds true for the planets.

Therefore if $\mathrm{D}=$ the mean density of the planet, $\frac{g c^{2} \mathrm{D}}{\mathrm{V}^{2} a d}=$ $\frac{25 \pi^{2} \times 2 \cdot 42}{3 \times 36}=5 \frac{\mathrm{I}}{2}$ nearly. Hence if $v=$ the velocity of propagation in the material which composes the nucleus of the earth, $\frac{g a}{v^{2}}=5 \frac{1}{2}$, or $v=\sqrt{\frac{2 g a}{11}}: v$ being calculated from this, is found to be $10 \cdot 13$ times the velocity of propagation in air:-a result far from being improbable. Generally, $\frac{g c^{2} \mathrm{D}}{\mathrm{V}^{2} a d}=\frac{g a}{v^{2}}$. Whence $\frac{\mathrm{V}}{v}=\frac{c}{a} \sqrt{\frac{\mathrm{D}}{d}}=\sqrt{\frac{c^{3} \mathrm{D}}{a^{3} d} \cdot \frac{a}{c}}$ $=\sqrt{\frac{m a}{\mathrm{M} c}}, m$ being the mass of the planet, $\mathrm{M}$ of the earth.

* I have there shown that, the velocity of uniform propagation $=\frac{\text { the velocity in the medium }}{\text { Naperian log. of the density }}$. This equation bears the same relation to the propagation of motion, as the equation, uniform velocity $=\frac{\text { space }}{\text { time }}$, to motion itself, and will not seem unimportant to those who consider that the one phxnomenon is nearly as frequent as the other. As the proposition is quite new, and in some degree contradicts the received mode of determining the velocity of propagation, it is not likely to meet with immediate attention: I have therefore adverted to it here. 
The following values of $\frac{V}{v}$, are calculated from the masses of the bodies of our system, as given by Laplace. For the Sun, 54.31; Jupiter, 5.33 ; Saturn, 3.06; Uranus, 1.97; the Earth, 1 ; Venus, $\cdot 981$; Mercury, $\cdot 651$; Mars, $\cdot 5$; the Moon, 23. It is observable that the order of magnitude of these quantities is the same as the order of the bodies arranged according to their masses. It follows, therefore, from our theory, that because in the greater masses the velocity of propagation is greater, the materials of which they are composed possess greater elastic force. This may be owing, not to any difference in the constituent elements, but to a greater degree of proper caloric, or of the force, whatever it be called, by which the constituent atoms are beld in their positions relatively to each other. For it is not unreasonable to suppose that the proper underived caloric of any mass, such as we know exists in the earth and forms the principal part of its caloric, is some function of the mass, and is specifically greater as the mass is greater. The Sun, which is the largest, is the hottest body of our system. According to this view, if there be granite at the surface of the moon, it will be more compressible than the granite of the earth; it will possess both a density and a compressibility depending on the mean density and compressibility of the moon's nucleus.

The theory we have been considering requires us to believe that the interior of a planet is solid, and not fluid. On the supposition of fluidity, it would be difficult to account for the contradiction presented by Mars to Clairaut's 'Theorem. May we not conjecture, that this planet is hollow about its centre, or in the direction of its axis? Generally speaking, the least bodies of the solar system are the densest, if we set aside the satellites, which seem to partake of the density of their primaries. But Mars is not so dense as Mercury, Venus, or the Earth. This fact favours in some degree the conjecture.

Assuming the truth of our theory, we may readily conceive that any change in the state of the internal heat of the earth, would give rise to great changes at its surface, and perhaps produce effects like those exhibited by geological phænomena.

Upon the whole, a review of the planets seems to favour the idea, that any increase of density towards their centres, is owing either wholly, or at least in part, to the compressibility of the matter of which their nuclei are composed.

Papworth, St. Everard, Aug. 10, 1831. 